Indonesian Journal of Physics

Vol 19 No. 4, October 2008

\title{
Determination of Energy Dependence Level Density Parameter and its Application in Fission Products Calculation of Pu-238
}

\author{
Abdul Waris ${ }^{1 *}$, Rizal Kurniadi ${ }^{1}$, Yuda S. Perkasa ${ }^{1}$, and Suwoto ${ }^{2}$ \\ ${ }^{1}$ Bosscha Laboratory, Department of Physics, \\ Faculty of Mathematics and Natural Sciences \\ Institut Teknologi Bandung, Bandung, Indonesia \\ ${ }^{2}$ Center for Reactor Technology and Nuclear Safety \\ National Nuclear Energy Agency of Indonesia, Serpong, Indonesia \\ *e-mail: awaris@fi.itb.ac.id
}

\begin{abstract}
The energy dependence of level density parameter has been calculated using extended wood-saxon potential. The extended version of potential has deep well parameter that is independent to the nucleon density. The potential is pure central interaction. Although the potential is different from mean field theory, it gives the better result than that of the reference input parameter library-2 (RIPL-2). The application of the level density parameter results in small discrepancies from ENDF results. This method has been adopted in example for calculating fission products of Pu238.
\end{abstract}

Keywords: Level density parameter, Extended wood-saxon potential, Fission products, $\mathrm{Pu}-238$

\section{Introduction}

In statistical mechanics calculation, such as evaporation model of nuclear reaction, spallation neutron measurements and studies of intermediateenergy heavy ion collision, level density plays an important role $^{1-3)}$. There are theoretical approaches have been developed to study the level density ${ }^{4-6)}$. Parameter that holds very important role in the level density is level density parameter ${ }^{7)}$. The commonly used of the level density parameter is an energy dependence parameter ${ }^{8-9)}$.

The asymptotic value of the level density parameter is reached at infinite excitation energy ${ }^{10)}$. Variation value is influenced by shell correction approach. Highly excitation state gives small variation of level density parameter values. The shell correction emerges as an effect of difference between the experimental nuclear mass and the semi empirical nuclear mass ${ }^{11)}$.

In the level density study, the semi empirical nuclear mass is influenced mainly by pair and shell correction. In the shell correction, fission barrier determines the variation of an eigen energy to smooth curve parts. In order to transfer the problem to become modest, the simple potential is usually chosen, such as an infinite square well or harmonic oscillator. The standard wood-saxon potential is one of the simple potential that commonly used for this purpose. However, the skin depth of this potential should be chosen randomly.

In order to overcome the later burden, an extended wood-saxon potential has been proposed for the present study. The extended wood-saxon potential is complex potential; hence the calculation needs numerical treatment. To reduce this complexity, the smooth curve calculation uses Gauss-Hermite folding technique $^{12)}$.

\section{Formulation}

The energy dependence of the level density is approximated by the formula ${ }^{13)}$ :

$$
a(A, Z, U)=\tilde{a}(A)\left\{1+\frac{\delta E}{U}(1-\exp (-\gamma U))\right\}
$$

$\tilde{a}$ parameter is the asymptotic value at infinite excitation energy $U$. There are three-semi empirical formulas of $\tilde{a}^{14)}$. In this paper the Iljinov formula has been chosen as the asymptotic parameter.

$$
\tilde{a}(A)=0.114 A+0.098 A^{\frac{2}{3}}
$$

$U$ is the value of excitation energy that was approximated by the following equation ${ }^{15)}$ :

$$
U=a_{C} t_{C}
$$

and $t_{C}$ is the critical temperature.

$$
\begin{aligned}
t_{C} & =0.567 \Delta \\
\Delta & =\frac{12}{\sqrt{A}}
\end{aligned}
$$

The semi empirical level density parameter $a_{C}$ has been proposed by Gilbert-Cameroon ${ }^{4}$.

$$
a_{C}=\frac{\pi^{2}}{6} g\left(e_{F}\right)
$$

with $g\left(e_{F}\right)$ is a single particle level density at fermi level that is $e_{F} \approx 33 \mathrm{MeV} \cdot g\left(e_{F}\right)$, which calculated by the following expression ${ }^{16)}$.

$$
g\left(e_{F}\right)=\frac{2 \sqrt{2}}{\pi \hbar^{2}} \int \sqrt{e_{F}-V(r)} r^{2} d r
$$

where the potential $V(r) \mathrm{s}$ the extended wood-saxon potential: 


$$
V_{\text {wS }}(r)=-\frac{V_{o}\left(1+\exp \left(-\frac{R}{d}\right)\right)}{\left(1+\exp \left(\frac{r-R}{d}\right)\right)}
$$

The potential is expanded through so-called the leptodermous approximation, hence equation (6) become

$$
V(r) \approx \begin{cases}V_{o}\left(1-e^{(r-R) / d}+e^{2(r-R) / d}\right) ; & r \leq r_{\lim } \\ 0 ; & r>r_{\lim }\end{cases}
$$

where $r_{\text {lim }}$ is the effective nuclear radius limit,

$$
r_{\text {lim }}=[R+\ln (0,618)] d
$$

Because of non-mean field approximation, the coulomb interaction uses the point to sphere technique $^{17)}$

$$
V_{\text {Coul }}(r)=\frac{Z_{1} Z_{2}}{R_{C}} f_{C}(x)
$$

where

$$
f_{C}(x)=\frac{1}{x} \tanh \left(\frac{3}{2} x+\frac{5}{8} x^{3}\right) ; R_{C}=R_{1}+R_{2} ; x=\frac{r}{R_{C}}
$$

then the interaction depicted by $V(r)=V_{W S}(r)+V_{\text {Coul }}(r)$, which replace the conventional one $V\left(\vec{r}_{1}\right)=\int V\left(\vec{r}_{12}\right) d^{3} \vec{r}_{2}$.

The iteration technique is applied to equation (1) to get the level density parameter ${ }^{18)}$.

$$
\begin{aligned}
& a_{0}=\tilde{a}(1+\delta E) \\
& U^{(n)}=a_{n} t_{C}^{2} \\
& a_{i+1}=\tilde{a}\left[1+\frac{\delta E}{U^{(n)}}\left(1-\exp \left(-U^{(n)}\right)\right)\right]
\end{aligned}
$$

$\delta E$ Parameter in equation (11) is so-called shell correction, which is calculated using formula below,

$$
\delta E=M_{\text {exp }}-M_{\text {Calc }}
$$

The experimental nuclear masses $M_{\text {exp }}$ are taken from AME2003 table ${ }^{19)}$ while $M_{\text {Calc }}$ is calculated from the binding energy formula,

$$
\begin{aligned}
B_{\text {nucl }}(A, Z)= & a_{v}\left(1-k_{v} I^{2}\right) A-a_{S}\left(1-k_{s} I^{2}\right) A^{2 / 3}-\frac{3}{5} \frac{e^{2} Z^{2}}{R_{o}}+E_{\text {pair }} \\
& -E_{\text {shell }}-a_{k} A^{1 / 3}-a_{o}-f_{p} \frac{Z^{2}}{A}-W|I|
\end{aligned}
$$

which all coefficients of equation (13) were compiled by Royer ${ }^{20)}$.

$$
\begin{aligned}
& \text { Where } \\
& E_{\text {pair }}= \begin{cases}-\frac{11}{\sqrt{A}} ; & Z, N: \text { odd } \\
0 \quad ; & \mathrm{A}: \text { odd } \\
\frac{11}{\sqrt{A}} ; & Z, N \text { :even }\end{cases}
\end{aligned}
$$

and $E_{\text {shell }}$ represents the shell correction, which is determined $\mathrm{by}^{21)}$ :

$$
E_{\text {shell }}=\int_{0}^{N}[\varepsilon(n)-\bar{\varepsilon}(n)] d n
$$

$\varepsilon(n)$ is an eigen energy, while $\bar{\varepsilon}(n)$ is smooth curve that was calculated from ${ }^{22)}$ :

$$
\int_{0}^{N} \bar{\varepsilon}(n) d n=\sum_{n=1}\left\{\begin{array}{l}
\frac{1}{2} \varepsilon_{n}\left(1+\operatorname{erf}\left(\bar{u}_{n}\right)\right)-\frac{1}{2 \sqrt{\pi}} \gamma e^{-\bar{u}_{n}^{2}}-\frac{1}{\sqrt{\pi}} e^{-\bar{u}_{n}^{2}} \\
\sum_{m=1} C_{m}\left[\frac{1}{2} \gamma H_{m}\left(\bar{u}_{n}\right)+\varepsilon_{n} H_{m-1}\left(\bar{u}_{n}\right)+m \gamma H_{m-2}\left(\bar{u}_{n}\right)\right]
\end{array}\right\}
$$

where,

$$
\bar{u}_{n}=\frac{\left(\bar{\lambda}-\varepsilon_{n}\right)}{\gamma}
$$

with fermi level $\bar{\lambda}$ that was obtained from the following relation:

$$
\bar{n}(\bar{\lambda})=N
$$

$N$ describes the nucleon number, where $\bar{n}(\varepsilon)$ has the following expression.

$$
\bar{n}(\varepsilon)=\sum_{n=1}\left\{\frac{1}{2}\left(1+\operatorname{erf}\left(u_{n}\right)\right)-\frac{1}{\sqrt{\pi}} e^{-u_{n}^{2}} \sum_{m=1} C_{m} H_{m-1}\left(u_{n}\right)\right\}
$$

with

$$
C_{m}=\left\{\begin{array}{l}
\frac{(-1)^{m / 2}}{2^{m}(m / 2) !} ; m: \text { even } \\
0
\end{array}\right.
$$

\section{Results and Discussion}

The extended wood-saxon potential has been applied in the level density parameter calculation. The Calculation that was obtained is compared with the reference input parameter library-2 (RIPL-2).

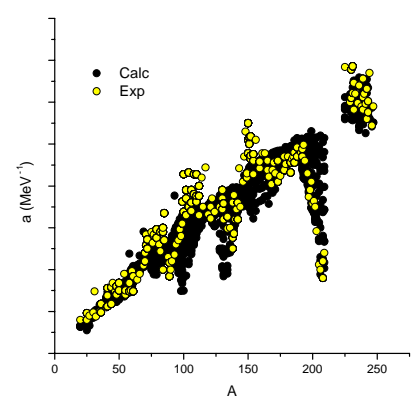

Figure 1. The calculated level density parameters (black) of $20<\mathrm{A}<250$ nuclei

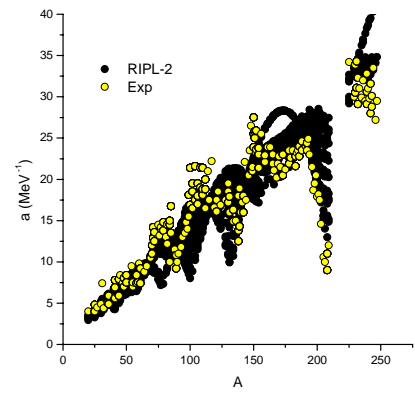


Figure 2. RILP-2 level density parameters (black) of 2 $<\mathrm{A}<250$ nuclei.

Figure 1 shows the level density parameters of the present work and Figure 2 illustrates the level density parameters of RIPL-2 result. These Figures clearly show that for $A=20$ to $A=150$ graphs of the level density parameters almost have similar pattern. Small discrepancies are taken place around $\mathrm{A}=75$ and between $\mathrm{A}=120$ up to $\mathrm{A}=150$ where the RIPL-2 results are higher than the experimental results

The peculiar of RIPL-2 results are taken place between $\mathrm{A}=150$ up to $\mathrm{A}=200$, where there are quadratic form in graph. The level density parameters of RIPL-2 have discrepancies significantly at that mass number range. Experimental results are lower than RIPL-2 at $\mathrm{A}=200$. The glare discrepancies between RIPL-2 and experimental one are showed at the range $\mathrm{A}=225$ up to $\mathrm{A}=250$.

The weakness of RIPL-2 results is overcome by using the extended wood-saxon potential. Figure.1 shows clearly that our calculation technique has results in lower values than that of RIPL-2 hence they have small discrepancies compared to the experimental one. Especially for $\mathrm{A}=125$ up to $\mathrm{A}=$ 250 , the present calculation gives best results.

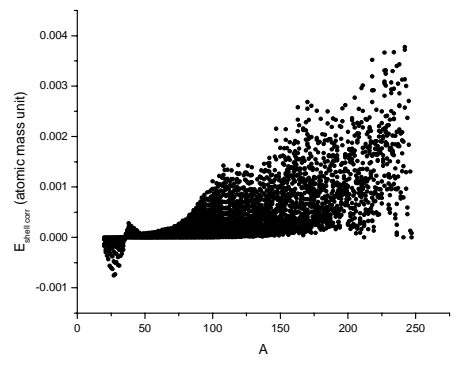

Figure 3 Extended wood-saxon shell correction of $20<$ A $<250$ nuclei

Figure 3 shows that the atomic mass number of above 125 gives the increasing of the shell correction rapidly hence equation (11) produces higher values. As a consequence, the increasing of shell correction value will trigger the decreasing of the level density parameter which finally results in our calculation give better results than that of RIPL-2.

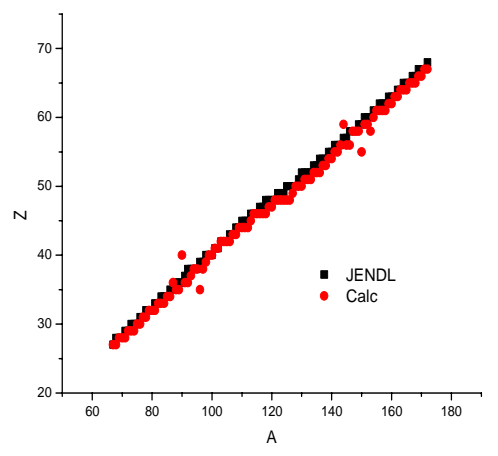

Figure 4. Fission product of n(Pu238,X)f

Implementation of the level density parameters result is for calculating the fission products of $\mathrm{Pu}-238$ as shown in Figure 4. Although there are small discrepancies about $A=95$ and $A=150$, generally the results are acceptable.

\section{Conclusion}

The extended wood-saxon potential has been employed for calculation the level density parameter of nuclide and gives the better results compared to that of RIPL-2 especially at mass number of above 150 . This potential is expanded through so-called the leptodermous approximation which is a skin depth approximation. This potential has been incorporated in calculating the fission products of $\mathrm{Pu}-238$ which results in a good agreement with the experimental one.

\section{Acknowledgment}

This research is fully funded by ITB research grant No. 0004/K01.03.2/PL2.1.5/I/2006, No 174/K01.07/PL/2007 and State Ministry of Research and Technology research grant No. 06/RD/Insentif/PPK/II/2008.

\section{References}

1. A. Waris et al., "Isotopic Fission-yield Calculation of U-233, U-238 and Th-232 for Fast Energy Spectrum", Indonesian Journal of Physics, 19:1, 9-32, January 2008.

2. Rizal Kurniadi and Abdul Waris, Calculation of Energy Dependent Level Density Parameter using Extended Wood-Saxon Potential, Proc. of the Intern. Conf. on Advances in Nuclear Science and Engineering 2007.

3. K. der Merr et al., Spallation Yields of Neutron Produced in Thick Lead/Bismuth Targets by Protons at Incident Energies of 420 and $590 \mathrm{MeV}$, Nucl Inst. And Meth. In Phys. Res. B 217, 202, 2004.

4. A. Gilbert, and A. G. W. Cameron, A. Composite Nuclear-Level Density Formula with Shell Corrections, Can. J. of Phys, 43, 1446, 1965.

5. S. Hilaire, Level Densities, Workshop on Nuclear Data and Nuclear Reactors : Physics, Design and Safety, 2000.

6. An $\mathrm{Yu}$, and $\mathrm{Hu}$ Jimin, Trial Densities for the Extended Thomas-Fermi Model, Nucl. Phys A 598, 1, 1995.

7. M. Ksczmarczyk, The Empirical Description of the Level Density Parameter $a$ in the Semiclassical Model, J. Phys. G : Nucl. Part. Phys, 26, 253, 2000.

8. P. Demetriou, and S. Goriely, Microscopic Nuclear Level Densities for Practical Applications, Nucl. Phys. A, 695, 95, 2001.

9. S. Hilaire, Energy Dependence of the Level Density Parameter, Phys. Lett. B 583, 264, 2004. 
10. D. Bucurescu and T. von Egidy, Systematic of Nuclear Level Density Parameters, J. of Phys. G : Nucl. Part. Phys. 31, S1675, 2005.

11. P. Moller, J. R. Nix, W. D. Myers, and W. J. Swiatecki, Nuclear Ground-state Masses and Deformations, At. Data and Nucl. Data. Tables, 59, 185, 1995.

12. B. Nerlo-Pomorska, K. Pomorski and J. Bartel (2006), Shell Energy and the Level Density Parameter of Hot Nuclei, Phys. Rev. C 74, 34327, 2006.

13. Handbook for Calculations of Nuclear Reaction data, RIPL-2, IAEA-TECDOC-1506, June 2006.

14. A. S. Iljinov, et al, Nucl. Phys. A 543, 5171992.

15. M. Rizea et al, Calculation of Nuclear Level Density Relevant for Thorium-Based Reactors, Rom. Rep. in Phys, 57:4, 757, 2005.
16. J. N. De, S. Shlomo, aad S. K. Samaddar, Level Density Parameter in a Refined Thomas-Fermi Theory, Phys. Rev. C, 57:3, 1398, 1998.

17. R. Anni, Analytical Approximation for the Sphere-Sphere Coulomb Potential, Phys. Rev. C 63, 067801, 2001.

18. M. Herman et al, EMPIRE Nuclear Reaction Model Code Ver 2.19 Lodi, March 2005.

19. G. Audi, A. H. Wapstra and C. Thibault, The AME2003 Atomic Mass Evaluation (II). Tables, Graphs and References, Nucl. Phys. A, 729, 337, 2003.

20. R. Royer, and C. Gautier, Coefficients and Terms of the Liquid Drop Model and Mass Formula, Phys. Rev. C, 73, 067302, 2006.

21. V. M. Strutinsky, Yad. Fis. 3, 614, 1966.

22. M. Bolsterli et al., New Calculation of Fission Barriers for Heavy and Superheavy Nuclei, Phys. Rev. C, 5, 1050, 1972. 